\title{
Global Complete Response in Viscera
}

National Cancer Institute

\section{Source}

National Cancer Institute. Global Complete Response in Viscera. NCI Thesaurus. Code C159964.

Liver or spleen or any org an considered involved at baseline should not be enlarged on physical exam and should be considered normal by imaging; no nodules should be present on imaging of liver or spleen; any post treatment mass must be determined by biopsy to be negative for lymphoma. 